\title{
Effects of Yinchenhao Tang and related decoctions on DMN-induced cirrhosis/fibrosis in rats
}

\author{
Cheng Liu ${ }^{1}$, Mingyu Sun ${ }^{1}$, Lei Wang1, Gaoqiang Wang1, Gaofeng Chen ${ }^{1}$, \\ Chenghai Liu ${ }^{1,2,3}$ and Ping Liu*1,2,3
}

\begin{abstract}
Address: ${ }^{1}$ Institute of Liver Diseases, Shuguang Hospital, Shanghai University of Traditional Chinese Medicine, Shanghai 201203, China, ${ }^{2}$ Shanghai University of Traditional Chinese Medicine, Shanghai 201203, China and ${ }^{3}$ E-institutes of Shanghai Municipal Education Commission, Shanghai University of Traditional Chinese Medicine, Shanghai 201203, China

Email: Cheng Liu - liucheng0082010@163.com; Mingyu Sun - mysun248@hotmail.com; Lei Wang - laoxia524@163.com; Gaoqiang Wang - wanggaoqiang2003@yahoo.com.cn; Gaofeng Chen - gaofengchen06@126.com; Chenghai Liu - chenghailiu@hotmail.com; Ping Liu* - liuliver@online.sh.cn

* Corresponding author
\end{abstract}

Published: 31 January 2008

Chinese Medicine 2008, 3:1 doi:10.1186/1749-8546-3-1
Received: 16 May 2007

Accepted: 31 January 2008

This article is available from: http://www.cmjournal.org/content/3/l/I

(C) 2008 Liu et al; licensee BioMed Central Ltd.

This is an Open Access article distributed under the terms of the Creative Commons Attribution License (http://creativecommons.org/licenses/by/2.0), which permits unrestricted use, distribution, and reproduction in any medium, provided the original work is properly cited.

\begin{abstract}
Background: Chinese medicine decoctions such as Yinchenhao Tang (YCHT), Xiayuxue Tang (XYXT), Huangqi Tang (HQT), Yiguan Jian (YGJ) and Xiaochaihu Tang (XCHT)) were used to treat liver cirrhosis. The present study evaluates the effects of these decoctions on fibrosis in rats induced by dimethylnitrosamine (DMN).

Methods: DMN solution ( $0.5 \%$ ) was injected to rats for three consecutive days per week for four weeks. At the beginning of week 3, rats were randomly divided into 4-week DMN control group, $Y C H T, X Y X T, H Q T, Y G], X C H T$ and vehicle groups. Each group was orally administered with specific decoctions daily for two weeks. Rats in the vehicle group were orally administered with only water.

Results: Liver fibrosis and cirrhosis were observed in weeks 2 and 4 in DMN-intoxicated rats. Compared with normal rats, alanine transaminase (ALT), aspartate transaminase (AST), alkaline phosphatase (ALP) activities and level of total bilirubin acid (TBA) in serum and content of Hydroxyproline (Hyp) in liver tissue of model group rats rose significantly. However, the albumin (Alb) level in serum decreased significantly. Compared with the 4-week DMN group, the pathological conditions and functions of the liver in the YCHT group improved significantly, and the content of Hyp decreased remarkably: only one rat in this group developed liver cirrhosis and the ratio of cirrhosis was only $8.3 \%$. On the other hand, the other decoctions did not show remarkable effects. YCHT inhibited $\alpha$-SMA activation, including its gene expression into mRNA and protein.
\end{abstract}

Conclusion: Among the five Chinese medicine decoctions, YCHT exerted the most significant therapeutic effects on DMN-induced cirrhosis/fibrosis in rats.

\section{Background}

Liver fibrosis occurs as a result of a variety of pathological factors, including viral hepatitis (especially hepatitis B and C), alcohol and drug abuse, metabolic diseases due to overload of iron or copper, autoimmunity against hepatocytes or bile duct epithelium, and congenital abnormali- 
ties [1]. Liver fibrosis, cirrhosis in particular, is associated with significant morbidity and mortality. As liver fibrosis may not manifest itself clinically until an advanced (i.e. cirrhotic) stage, the capability of reversing the fibrosis is highly desirable for developing therapeutic approaches [2]. At present, there is no standard treatment for liver fibrosis. Experimental studies indicated that the progression of liver fibrosis can be prevented in rodents [3], although the efficacy of most of the preventive treatments has not been established in humans. Therefore, it is imperative to develop anti-fibrotic strategies to treat liver fibrosis and cirrhosis.

Chinese medicine has been widely used for treating chronic liver hepatitis and liver cirrhosis. Chinese medicine treatment improved clinical symptoms, liver function and quality of life for patients, inhibited liver inflammation and fibrous tissue proliferation, and reversed liver fibrosis. According to Chinese medicine theories, liver cirrhosis is caused by internal damp (Shi) heat $(R e)$, blood (Xue) stasis, and both $Q i$ and Yin asthenia. Thus, the main Chinese medicine approach to liver cirrhosis is to eliminate heat, dispel dampness, activate blood, promote Qi and cultivate Yin [4-6]. In this study, five classical decoctions, namely Yinchenhao Tang (YCHT), Xiayuxue Tang (XYXT), Huangqi Tang (HQT), Yiguan Jian (YGJ) and Xiaochaihu Tang (XCHT) were used to treat liver cirrhosis induced by DMN (dimethylntrosamine) in rats.

First described in Shanghan Lun in $200 \mathrm{AD}$, YCHT consists of three medicinal herbs: Herba Artemisiae Scopariae (Yinchenhao), Fructus Gardeniae (Zhizi), and Radix et Rhizoma Rhei (Dahuang), at a weight ratio of 18:10:6 respectively. YCHT has been recognized by some practitioners and researchers for its beneficial effects in treating liver diseases [7]. YCHT was shown to ameliorate hepatitis [8] and liver fibrosis in various animal fibrotic models [9-12]. XYXT was first described in Jinkui Yaolue in the East Han Dynasty. It consists of three herbs: Radix et Rhizoma Rhei (Dahuang), Semen Persicae (Taoren), and Eupolyphaga Seu Steleophaga (Tubiechong), at a weight ratio of 10:10:6 respectively. XYXT significantly inhibited liver fibrosis induced by porcine serum [13] and $\mathrm{CCl}_{4}$ in rats. $H Q T$ was first described in Hejiju Fang. It consists of three medicinal herbs: Radix Astragali (Huangqi), Radix et Rhizoma Glycyrrhizae (Gancao), and Fructus Jujubae (Dazao), at a weight ratio of $6: 1: 1$. HQT and its components inhibited bileduct-ligation (BDL)-induced cholestatic cirrhosis [14]. YGJ was first described in Liuzhou Yihua and consists of six herbs: Radix Glehniae (Beishashen), Radix Ophiopogonis (Maimendong), Radix Angelicae Sinensis (Danggui), Radix Rehmanniae (Shengdihuang), Fructus Lycii (Gouqizi), and Fructus Toosendan (Chuanlianzi). It was found to inhibit $\mathrm{CCl}_{4}$-induced cirrhosis [15]. XCHT consists of seven herbs: Radix Bupleuri (Chaihu), Radix Scutellariae (Huang-
qin),Radix Ginseng (Renshen),Rhizoma Pinelliae (Banxia),Radix et Rhizoma Glycyrrhizae (Gancao),Rhizoma Zingiberis Recens (Shengjiang),Fructus Jujubae (Dazao). It was first described in Shanghan Lun and has been widely used to treat chronic hepatitis in Japan since the 1970s. Its anti-fibrosis action has been reported [16].

This study aims to (1) determine which of these five decoctions is most effective to treat DMN-induced liver fibrosis; and (2) establish a molecular basis of the most effective decoction.

\section{Methods}

\section{Chemicals and herbs}

DMN was purchased from Sigma. Hydroxyproline (Hyp) standard was purchased from Nakateitesuku Corporation (Japan). Liver function test kit was purchased from Nanjing Jiancheng Biotech Company (China). XCHT (TJ-9) was purchased from Tumura Corporation (Japan). Herb constituents of YCHT, XYXT, HQT and YGJ were purchased from Shanghai Huayu Chinese Herbs Co Ltd (China). The herbs were accredited by a pharmacognosist according to standard protocols, prepared by Shuguang Hospital, and then stored at $-20^{\circ} \mathrm{C}$.

\section{Animal models of liver fibrosis}

Eighty-eight male Wister rats of weight 180-200 g were kept in an air-conditioned room at $25^{\circ} \mathrm{C}$ with a 12 hour dark/light cycle and unlimited access to food and water. They were randomly divided into two groups: (1) control $(\mathrm{n}=10)$ and (2) DMN ( $=78)$. DMN (10 mg/kg body weight) was administered intraperitoneally to DMN rats for three consecutive days each week for four weeks [2]; normal saline was given to control rats. At the end of week 2, three control rats and six DMN rats were sacrificed for observation. The remaining DMN rats $(\mathrm{n}=72)$ were randomly divided into six groups: $\mathrm{DMN}+$ water, YCHT, XYXT, HQT, YGJ and XCHT $(\mathrm{n}=12)$. At the end of week 4 , all rats were anesthetized with $40 \mathrm{mg} / \mathrm{kg}$ body weight sodium pentobarbital. Blood was collected from interior vena cava. The livers and spleens were immediately removed, washed with ice-cold saline, dried by filter paper, and weighed in the wet state.

\section{Liver function tests}

Serum was collected from blood by centrifugation at 1300 $\times$ g at $4^{\circ} \mathrm{C}$ and stored at $-80^{\circ} \mathrm{C}$ for liver function tests. Serum activities of alanine transaminase (ALT), aspartate transaminase (AST), alkaline phosphatase (ALP), content of albumin (Alb) and total bilirubin (TBIL) were tested. Total bile acid (TBA) was measured by a clinical laboratory of Shuguang Hospital. 


\section{Histology examination}

Liver tissues were taken from the right lobe of the liver of each rat, and fixed in $4 \%$ buffered paraformaldehyde, dehydrated in a graded alcohol series. Following xylene treatment, specimens were embedded in paraffin blocks, cut into $5 \mu \mathrm{m}$-thick sections and placed on glass slides. The sections were stained with hematoxylin-eosin and Sirius red respectively. Fibrosis was graded according to the method by Scheuer [17] as follows: grade 0: normal liver; grade 1: an increase of collagen without the formation of septa (small satellite expansion of the portal fields); grade 2: formation of incomplete septa not interconnecting with each other, from the portal tract to the central vein; grade 3: complete but thin septa interconnecting with each other, which divide the parenchyma into separate fragments; and grade 4: complete cirrhosis, similar to grade 3 with thicker septa. The pathological examination was performed by three pathologists who were blinded to the rats' treatment assignment. Fibrosis scores were given after the pathologists had thoroughly examined three different areas of the tissue slide for each rat.

\section{Hepatic hydroxyproline content}

Liver tissue (100 mg) was prepared for hydroxyproline (Hyp) determination according to a modification of method by Jamall et al. [18]. The Hyp content of the liver as an indirect measure of tissue collagen content was expressed as microgram per gram of wet weight $(\mu \mathrm{g} / \mathrm{g})$.

\section{Expression and immunochemistry of $\alpha$-SMA}

Total RNA was extracted from $50 \mathrm{mg}$ of liver tissue using Trizol reagent (Invitrogen, USA). Synthesis of cDNA was performed using $4 \mu \mathrm{g}$ of total RNA per sample with random primers and reagents contained in the RevertAid First Strand cDNA synthesis kit (Fermentas, Lithuania). Two microliters $(2 \mu \mathrm{l})$ of each sample was used for real-time PCR in a Rotor-Gene 2000 system (Australia). Relative quantitation was calculated using delta cycle threshold (CT) relative quantitation. Sequences and accession number of primers used for alpha smooth muscle actin $(\alpha$-SMA) are: 5'-CGAGAGGACGTTGTTAGCATAGAG-3' (forward) and 5'-GGGCATCCACGAAACCA-3' (reverse) (GenBank accession number: BI282702; product length: $85 \mathrm{bp}$ ). Sequences and accession number of primers used for 18S rRNA are: 5'-GTAACCCGTTGAACCCCATT-3' (forward) and 5'-CCATCCAATCGGTAGTAGCG-3' (reverse) (GenBank accession number: X00117; product length: $151 \mathrm{bp})$.

Liver samples were prepared with Radio immunoprecipitation assay (RIPA) lysis buffer containing protease inhibitors at $4{ }^{\circ} \mathrm{C}$. After quantification, the proteins $(50 \mu \mathrm{g} /$ lane) were separated by polyacrylamide gel electrophoresis and transferred to Immobilon-P membranes which were then blocked and exposed to $\alpha$-SMA (Chemicon,
CBL171, 1:100, USA). The antigen was demonstrated by enhanced chemiluminescence (Pierce, USA) for one minute, followed by exposure to Kodak film (Kodak, USA).

After deparaffinization and dehydration, microwave antigen retrieval was performed for five minutes prior to peroxidase quenching with $3 \% \mathrm{H}_{2} \mathrm{O}_{2}$ in PBS for 15 minutes. Subsequently, the sections were preblocked with 5\% bovine serum albumin for 30 minutes. Slides were incubated with anti- $\alpha$-SMA (Sigma, A2547, 1:400, USA) antibody overnight at $4{ }^{\circ} \mathrm{C}$ and with biotinylated secondary antibodies for 45 minutes respectively. They were then developed with diaminobenzidine (DAB) for 3 minutes and finally counterstained with hematoxylin.

\section{Statistical analysis}

All results were expressed as mean and standard deviation (SD). Measurement data were analyzed using a one-way analysis of variances (ANOVA, SPSS 11.0). Rank data were analyzed with ridit. The result of $\mathrm{P}<0.05$ was considered to be statistically significant.

\section{Results}

\section{Effects on survival and liver/spleen weight ratio}

During drug intervention process, two rats died in the $\mathrm{DMN}+X C H T$ group. Animal body and liver weights were monitored during the formation of liver fibrosis (Table 1 ). Body weights of the rats from the 2-week DMN group and 4-week DMN + water group were significantly lower than those of the control group during the same period ( $P$ $<0.01)$. Livers of the control rats were smooth, ruddy, and soft, while those of the 2-week DMN rats were slightly bigger, accompanied by hyperemia and edema. In the DMN + water rats, livers were much smaller and more rigid. Liver/body weight ratio of the $\mathrm{DMN}+$ water group was significantly lower than that of the control group $(\mathrm{P}<$ 0.01); spleen/body weight ratio of the $\mathrm{DMN}+$ water group significantly increased $(\mathrm{P}<0.01)$. The liver/body weight ratio of the YCHT group was higher than that of the $\mathrm{DMN}+$ water group without significant difference. Spleen weight of the YGJ group was significantly lower than that of the 4-week DMN control group $(\mathrm{P}<0.05)$. No significant improvements were found in the other groups.

\section{Effects on hepatic histopathological changes}

Livers in the control group showed normal lobular architecture with central vein and radiating hepatic cords (Figure 1a). After two weeks of DMN-intoxication, massive hepatocyte necrosis, intense neutrophilic infiltration, mild bile duct hyperplasia and initiation of fibrosis were found (Figure 1a). There was diffuse centrilobular congestion with marked dilatation of central veins. Extensive necrosis and hemorrhage were prominent at this early 
Table I: Effects of five Chinese medicine decoctions on body weight, liver weight, spleen weight, liver/body, spleen/weight in DMN induced liver fibrosis of rats

\begin{tabular}{lllllll}
\hline Group & Group size (n) & Body weight(g) & Liver weight (g) & Spleen weight (g) & Liver/body (\%) & Spleen/body (\%o) \\
\hline 2-week control & 3 & $226.0(2.64) \triangle \triangle$ & $7.80(0.26) \triangle \triangle$ & $0.66(0.07) \triangle \triangle$ & $3.45(0.12) \triangle \triangle$ & $2.90(0.28) \triangle \triangle$ \\
2-week DMN & 6 & $175.8(6.11)$ & $7.02(0.32)$ & $1.02(0.12)$ & $3.99(0.09)$ & $5.80(0.58)$ \\
4-week control & 7 & $287.0(8.98) \triangle \triangle$ & $10.03(0.49) \triangle$ & $0.76(0.05) \triangle \triangle$ & $3.49(0.08) \triangle \triangle$ & $2.64(0.17) \triangle \triangle$ \\
DMN + water & 12 & $204.2(20.22)$ & $5.69(1.78) \triangle$ & $1.43(0.22) \triangle \triangle$ & $2.73(0.61) \triangle \triangle$ & $7.27(0.35) \triangle$ \\
DMN + YCHT & 12 & $197.27(22.10)$ & $5.91(1.95)$ & $1.49(0.25)$ & $2.93(0.74)$ & $7.54(0.68)$ \\
DMN + XYXT & 12 & $208.33(15.11)$ & $5.22(1.42)$ & $1.60(0.21)$ & $2.48(0.54)$ & $7.67(0.66)$ \\
DMN + HQT & 12 & $212.00(20.20)$ & $5.52(1.70)$ & $1.57(0.20)$ & $2.58(0.72)$ & $7.44(0.87)$ \\
DMN + YG & 12 & $193.17(24.41)$ & $4.50(1.57)$ & $1.29(0.21)$ & $2.28(0.59)$ & $6.65(0.67)$ \\
DMN + XCHT & 10 & $216.70(17.08)$ & $5.22(1.16)$ & $1.55(0.15)$ & $2.40(0.42)$ & $7.17(0.72)$ \\
\hline
\end{tabular}

$P<0.05 P<0.01$ (vs DMN+ water)

$\triangle \mathrm{P}<0.05 \triangle \triangle \mathrm{P}<0.0 \mathrm{I}$ (vs 2-week $\mathrm{DMN}$ )

YCHT: Yinchenhao Tang

XYXT: Xiayuxue Tang

HQT:Huangqi Tang

YG): Yiguang Jian

XCHT: Xiaochaihu Tang

Results are expressed as mean (SD).

stage. In the DMN + water group, the liver sections revealed collagen deposition, marked cirrhosis, collagen fiber deposition, severe centrilobular necrosis, focal fatty changes, bile duct proliferation, bridging necrosis and apoptosis, and fibrosis surrounding the central veins (Figure 1a). In the DMN + YCHT group, marked reduction of the thickening of the collagen bundles was seen. In the $\mathrm{DMN}+\mathrm{XYXT/HQT/YGJ/XCHT}$ groups, changes in hepatocyte necrosis and bile duct hyperplasia were shown (Figure 1a).

\section{Effects on liver collagen deposition}

Quantitative assessment of liver fibrosis was performed with morphometry on sections processed with $0.1 \%$ Sirius red which specifically stains collagen. Collagen was scarcely observed except around the small central venous walls in normal liver (Figure 1b). In the 2-week DMN rats, collagen was seen to stretch from portal area to lobular, and incomplete septa were also observed (Figure 1b). In the DMN + water rats, livers showed marked distortion in architecture, including portal and lobular bridging fibrosis, cirrhotic nodule formation, and thickened reticulum fibers joining central areas. Normal structure disappeared, while pseudolobules formed (Figure 1b) with 75\% rats with cirrhosis. Compared with the DMN + water group, fibrosis scores of livers in YCHT group significantly decreased $(\mathrm{P}<0.05)$. Only one rat developed typical cirrhosis; the rate of cirrhosis was $8.3 \%$. Ridit analysis showed that the DMN + YCHT rats were significant different from the DMN + water rats $(\mathrm{P}<0.05)$. Other intervention groups were not significant different (Figure 1 and Table 2).

\section{Effects on hepatic hydroxyproline content}

Changes in Hyp content in the liver are considered an index for collagen metabolism and provide valuable information about the biochemical and pathological states of liver fibrosis. Liver collagen content, expressed as microgram $(\mu \mathrm{g})$ of Hyp/gram $(\mathrm{g})$ of liver tissue, is shown in Table 2. The Hyp content in the DMN rats was approximately $161 \%$ of that of the control group $(\mathrm{P}<0.01)$ at the end of week 2, suggesting abundant accumulation of collagen in the livers of DMN rats. The highest increase, which was about 3.6-fold compared with the control $(\mathrm{P}<$ 0.01 ), was observed at the end of week 4 in the DMN rats + water group. It was consistent with the observation of marked cirrhosis and accumulation of collagen bundles in the liver by histopathological examination. There was a significant decrease $(P<0.05)$ in liver Hyp content (about $41 \%$ in the DMN + water rats) in the YCHT rats, suggesting that YCHT ameliorated hepatic collagen deposition in DMN-induced liver injury. Hyp content in the DMN + HQT/XYXT rat livers was slightly reduced without significant difference. Hyp content in DMN + XCHT/YGJ rats was not changed.

\section{Effects on liver function}

Liver function parameters deteriorated over time in rats subjected to DMN (Table 1). In DMN rats, ALT, AST, ALP, TBIL and TBA levels were significantly higher after two and four weeks compared with the control rats $(\mathrm{P}<0.01)$, indicating hepatic injury. ALT, AST, ALP, TBIL and TBA levels also increased markedly in the cirrhotic DMN + water rats. Oral administrations of YCHT reduced the DMN-induced increase in ALT, AST, ALP activities as well as TBIL and TBA content $(P<0.01)$. These decreased biochemical parameters in the DMN + water rats indicated 


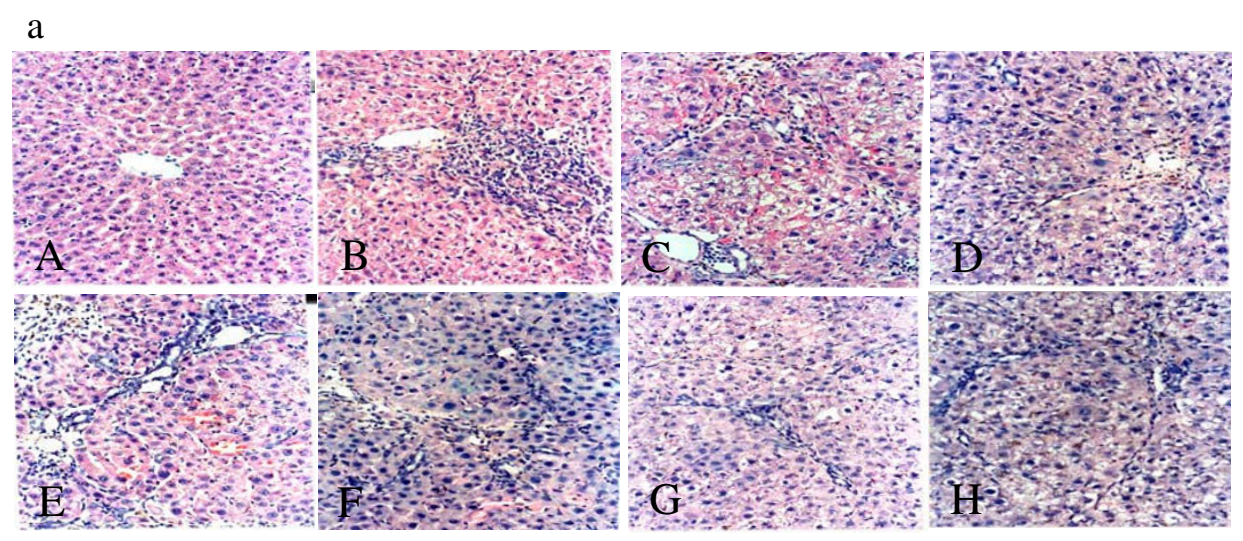

$\mathrm{b}$

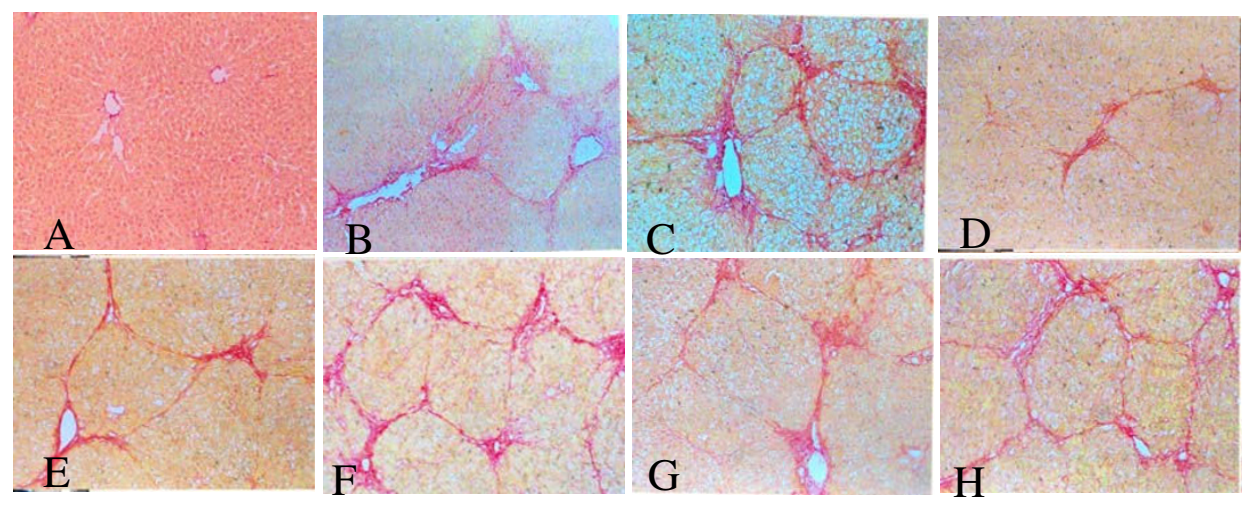

\section{Figure I}

Effects of the five Chinese medicine decoctions on histological changes of liver. (a) Hematoxylin - eosin (H\&E) $\times 200$. (A) Control rats (B) 2-week DMN fibrotic rats (C) DMN + water cirrhotic rats (D) DMN + YCHT rats (E) DMN + XYXT rats (F) DMN $+H Q T$ rats $(G) D M N+Y G$ rats $(H) D M N+X C H T$ rats. (b) Sirius red stain $\times 100$. (A) Collagen was scarcely observed. (B) Collagen was stretched from portal to lobular area. (C) Portal and lobular bridging fibrosis, and cirrhotic nodule formation were observed. (D) Fibrosis significantly decreased. (E), (F), (G) and (H) Changes in collagen deposition were observed.

that YCHT ameliorated hepatic injury in the DMN rats. Serum Alb content in the 2-week and 4-week DMN rats was significantly lower than that in the control rats. YCHT could elevate Alb content, but there was no significant difference compared with the DMN + water group (Table 3 ).

\section{Effects of YCHT on the gene expression of $\alpha-S M A$ into mRNA and protein}

In liver function test, histopatholgical observation and hepatic Hyp content detection, YCHT showed a comprehensive inhibitory effect on DMN-induced liver fibrosis. We went on to detect the anti-fibrotic function of YCHT using molecular biology techniques.

Levels of $\alpha$-SMA mRNA increased dramatically in the DMN rats at week $2(\mathrm{P}<0.01)$ (Figure $2 \mathrm{a})$. They also increased remarkably in the DMN + water group $(\mathrm{P}<$ $0.01)$. In the DMN + YCHT group, $\alpha$-SMA mRNA expression decreased remarkably $(\mathrm{P}<0.05)$ compared with the
DMN + water group. The result was also confirmed by immunoblotting (Figure 2b).

In the control rats, $\alpha$-SMA-positive hepatic stellate cells (HSCs) were weakly stained and were only detected in vascular structures (Figure 2c). Along with DMN-intoxication, the number of $\alpha$-SMA-positive HSCs increased gradually and reached peak at week 4 in cirrhotic livers (Figure $2 c)$. In the DMN + YCHT group, number of $\alpha$-SMA-positive HSCs was significantly reduced (Figure 2c).

\section{Discussion}

DMN-induced liver fibrosis in rats is a reproducible model for studying the pathogenesis of liver fibrosis and cirrhosis $[19,20]$. DMN-intoxication for four weeks produced centrilobular necrosis and fully-developed cirrhosis in rats. The approximately four-fold increase of total liver collagen observed in the present study is consistent with the previous investigations on DMN-induced liver fibrosis 
Table 2: Effects of five Chinese medicine decoctions on liver hydroxyproline (Hyp) content in DMN-induced liver fibrosis of rats

\begin{tabular}{|c|c|c|c|c|c|c|c|c|}
\hline \multirow[t]{2}{*}{ Group } & \multirow[t]{2}{*}{ Group size (n) } & \multirow[t]{2}{*}{ Hyp content $\mu g / g$ mean (SD) } & \multicolumn{5}{|c|}{ Fibrotic grade* } & \multirow[t]{2}{*}{$95 \% \mathrm{Cl}$ of $\mathrm{U}^{* *}$} \\
\hline & & & 0 & 1 & 2 & 3 & 4 & \\
\hline Control & 10 & $179.36(9.90)$ & 10 & 0 & 0 & 0 & 0 & $(-0.110$ to 0.228$)$ \\
\hline 2-week DMN & 6 & $289.06(26.95)$ & 0 & 0 & 4 & 2 & 0 & $(0.05 I$ to 0.486$)$ \\
\hline DMN + water & 12 & $64 I .71(54.22) \triangle \triangle$ & 0 & 0 & 0 & 3 & 9 & (0.586 to 0.894$) \triangle \triangle$ \\
\hline$D M N+Y C H T$ & 12 & $434.16(25.39)$ & 0 & 0 & 5 & 6 & I & (0.226 to 0.548$)$ \\
\hline $\mathrm{DMN}+X Y X T$ & 12 & $586.91(37.53)$ & 0 & 0 & 0 & 5 & 7 & (0.522 to 0.829$)$ \\
\hline $\mathrm{DMN}+\mathrm{HQT}$ & 12 & $559.04(47.06)$ & 0 & 0 & 0 & 8 & 4 & (0.580 to 0.734$)$ \\
\hline$D M N+Y G J$ & 12 & $647.17(64.73)$ & 0 & 0 & 1 & 6 & 5 & (0.435 to 0.743$)$ \\
\hline $\mathrm{DMN}+\mathrm{XCHT}$ & 10 & $636.35(48.53)$ & 0 & 0 & I & 7 & 2 & (0.333 to $0.67 I)$ \\
\hline
\end{tabular}

*: grade 0: normal; grade I: very slight; grade 2: slight; grade 3: moderate; grade 4: severe. Data are expressed as numbers of animals with each fibrotic grade.

**: $95 \%$ confidence interval of the $U$ values obtained from ridit analysis

$\mathrm{P}<0.05 \mathrm{P}<0.01$ (vs DMN+ water)

$\triangle P<0.05 \triangle \triangle P<0.0$ I (vs 2-week DMN)

YCHT: Yinchenhao Tang

XYXT: Xiayuxue Tang

HQT:Huangqi Tang

YG]: Yiguang Jian

XCHT: Xiaochaihu Tang

Results are expressed as mean (SD).

in rats [21-24]. Two weeks of DMN-intoxication produced diffuse centrilobular congestion with marked dilatation of central veins. Extensive necrosis and hemorrhage were prominent at this early stage; all rats developed liver fibrosis of grades 2-3. Liver Hyp content significantly increased 1.61-fold as compared with the normal rats. Notable abnormality in liver function was also observed. In the $\mathrm{DMN}+$ water group, liver damage significantly increased; liver weight notably reduced and splenomegaly was observed. Liver structure became disordered seriously with rapid progress of fibrosis [25-27]. Hyp content in liver tissue of the DMN + water group, which increased 3.68-fold, was much higher than that of the control group. Seventy-five per cent (75\%) of the DMN + water group have developed cirrhosis. This study related to the common symptoms of liver cirrhosis as well as its pathogenesis in traditional Chinese medicine. Five decoctions were chosen to prevent the rapid formation phase of liver cirrhosis from fibrosis. This study revealed different efficacies of the five decoctions. The results showed that YCHT had some specific therapeutic effects on pathological changes in the liver. Liver weight reduction was stopped and liver function was improved; serum activities of ALT, AST and ALP were restrained at basic level for the 2-week DMN rats; serum TBIL content was lowered for 2-week DMN rats. Liver cell degeneration, necrosis, inflammation

Table 3: Effects of five Chinese medicine decoctions on serum activities of AST, ALT and ALP and serum content of TBA and TBIL in DMN-induced liver fibrosis of rats

\begin{tabular}{|c|c|c|c|c|c|c|}
\hline Group & $\mathrm{ALT}(\mathrm{U} / \mathrm{L})$ & $\mathrm{AST}(\mathrm{U} / \mathrm{L})$ & ALP $(K / / 00 \mathrm{ml})$ & $\mathrm{TBA}(\mu \mathrm{mol} / \mathrm{L})$ & TBIL $(g / L)$ & Alb (g/L) \\
\hline Control & $20.13(3.10) \triangle \triangle$ & $28.03(4.33) \triangle \triangle$ & $33.73(4.95) \triangle \triangle$ & $19.30(6.03) \triangle \triangle$ & $11.5(0.93) \triangle \triangle$ & 40.89 (2.23) $\triangle \triangle$ \\
\hline 2 week DMN & $40.62(4.96)$ & $42.08(2.35)$ & $56.85(9.80)$ & $23.5(8.06)$ & $20.0(6.52)$ & $35.06(2.27)$ \\
\hline $\mathrm{DMN}+$ water & 70.30 (6.38) $\triangle \triangle$ & $81.08(3.18) \triangle \triangle$ & 97.67 (3.58) $\triangle \triangle$ & $56.8(9.45) \triangle \triangle$ & $24.1(7.71) \triangle \triangle$ & $26.38(4.11) \triangle \triangle$ \\
\hline $\mathrm{DMN}+\mathrm{YCHT}$ & $39.34(5.87)$ & $45.39(5.16)$ & $77.20(5.28)$ & $38.1(10.08)$ & $16.7(4.68)$ & $27.06(4.16)$ \\
\hline$D M N+X Y X T$ & $60.26(6.70)$ & $70.89(5.72)$ & $83.55(13.26)$ & $38.0(12.90)$ & $16.9(6.68)$ & $26.74(4.7 I)$ \\
\hline $\mathrm{DMN}+\mathrm{HQT}$ & 58. $23(0.80)$ & $66.12(5.58)$ & $94.19(6.35)$ & $40.8(10.47)$ & $19.0(2.36)$ & $28.89(2.20)$ \\
\hline$D M N+Y G J$ & $66.77(5.29)$ & $75.09(2.25)$ & $79.37(3.39)$ & $47.0(21.02)$ & $20.0(15.19)$ & $26.13(3.67)$ \\
\hline $\mathrm{DMN}+X \mathrm{XCHT}$ & $68.41(5.82)$ & 77.42 (5.59) & $93.38(1.98)$ & $55.3(14.34)$ & $23.0(13.52)$ & $25.88(2.95)$ \\
\hline
\end{tabular}

$P<0.05 P<0.01$ (vs DMN+ water)

$\triangle \mathrm{P}<0.05 \triangle \triangle \mathrm{P}<0.0 \mathrm{I}$ (vs 2-week $\mathrm{DMN}$ )

YCHT: Yinchenhao Tang

XYXT: Xiayuxue Tang

HQT:Huangqi Tang

YG): Yiguang Jian

XCHT: Xiaochaihu Tang

Results are expressed as mean (SD). 
a

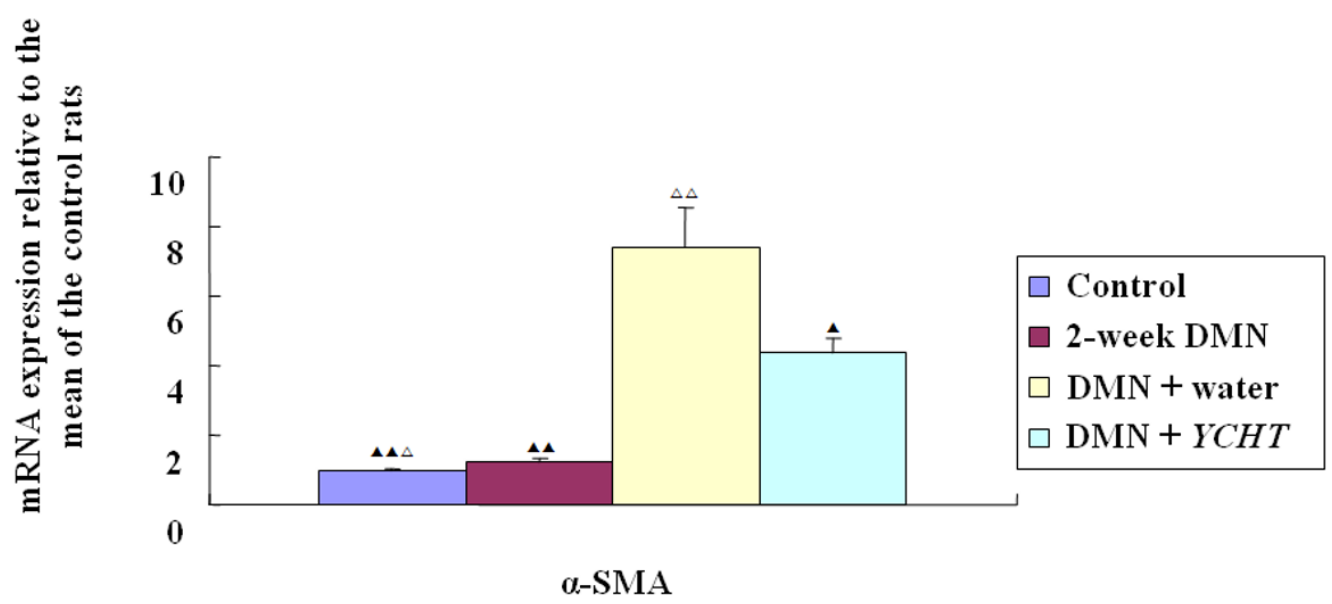

b

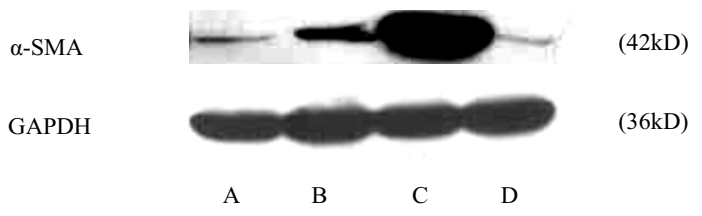

c
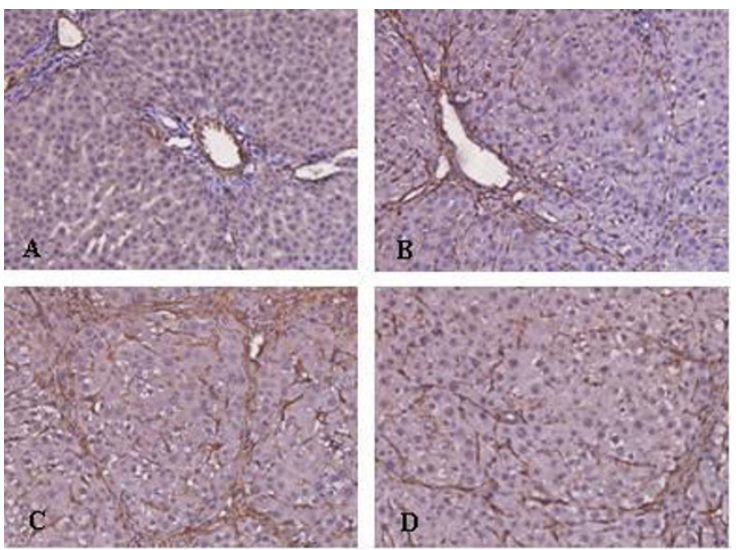

Figure 2

(a) Real-time quantitative PCR. In the 2-week DMN group, $\alpha$-SMA mRNA expression level increased (vs control rats, $P<$ $0.0 \mathrm{I})$. In the DMN + water group, $\alpha$-SMA mRNA expression level also increased (vs control rats, $P<0.0 \mathrm{I}$ ). In the DMN + $Y C H T$ group, $\alpha$-SMA mRNA expression level decreased (vs DMN + water rats, $\mathrm{P}<0.05) . \mathrm{P}<0.05 \quad \mathrm{P}<0.0 \mathrm{I}$ (vs $\mathrm{DMN}+$ water) $\triangle P<0.05 \triangle \triangle P<0.0$ I (vs 2-week DMN). (b) Western blot. Western blot analysis of total protein extracts was performed with antibodies recognizing $\alpha$-SMA and Glyceraldehyde 3-phosphate dehydrogenase (GAPDH). YCHT reduced $\alpha$-SMA protein expression significantly (vs DMN + water). (c) Immunohistochemical analysis $\times 200$. (A) In the control group, $\alpha$-SMA-positive HSCs were weakly stained and only detected in vascular structures. (B) and (C) With DMN-intoxication, number of $\alpha$-SMApositive HSCs increased gradually and peaked at week 4. (D) In the DMN + YCHT group, number of $\alpha$-SMA-positive HSCs was significantly reduced. 
and infiltration were significantly reduced. Liver fibrous tissue hyperplasia and liver structural alterations were markedly inhibited. Only one of the 12 rats had liver cirrhosis, cirrhosis formation rate being $8.3 \%$. Hyp content was $44.9 \%$, lower than that of the $\mathrm{DMN}+$ water group. The other four decoctions did not show these significant effects. Hepatocyte necrosis, sinusoidal endothelial injury, inflammation and bleeding induced by DMN were considered to be the major factors for the development of liver fibrosis. Sustained inflammation caused extracellular matrix deposition and nodular regenerative transition $[16,28]$.

Activation of HSCs is a key factor in developing liver fibrosis. Inhibition of the accumulation of activated HSCs by modulating either their activation and/or proliferation or promoting their apoptosis is thus an important therapeutic strategy. YCHT reduced $\alpha$-SMA (a marker of activated HSCs [29]) expression at mRNA and protein synthesis levels. Our results indicated that the molecular mechanism of anti-fibrotic effects by $\mathrm{YCHT}$ might be due to the suppression of HSCs' activation.

In another study by our team, we used the same decoctions for treating liver cirrhosis induced by DMN in rats at the cirrhosis formation stage. The rats were injected with DMN for four weeks and were treated with the herbal medicines for two weeks. Among the five decoctions, both $Y C H T$ and HQT exerted significant therapeutic effects and YCHT was overall more effective than HQT [29]. The present study demonstrated the effectiveness of YCHT in $\mathrm{DMN}$-induced rat liver fibrosis/cirrhosis and pathological changes in different stages of the disease. In this study, rats were exposed to DMN and received herbal medicine intervention after the formation of liver fibrosis after two weeks of DMN-intoxication. In general no significant effect of intervention by HQT was found; although it was previously found to be effective in DMN-induced liver cirrhosis. It is probable that the rats which were exposed to DMN sustained as long as intervention therapy was given. However, when liver cirrhosis was formed and pathogenic factors were removed for two weeks, inflammatory injury could be relaxed gradually. Hepatic parenchymal cell injury appears gradually in this period. Thus, pathological changes in various stages of liver fibrosis and cirrhosis may affect the efficacy of the decoctions.

\section{Conclusion}

Among the five Chinese medicine decoctions, YCHT demonstrated the most significant anti-fibrotic effects on DMN-induced liver cirrhosis/fibrosis in rats, which may be a result of the decoction's inhibitory effects on HSCs' activation.

\section{Abbreviations}

$\alpha$-SMA: $\alpha$-smooth muscle actin; Alb: albumin; ALP: alkaline phosphatase; ALT: alanine transaminase; AST: aspartate transaminase; CT: cycle threshold; DAB: diaminobenzidine; DMN: dimethylnitrosamine; HSCs: hepatic stellate cells; Hyp: hydroxyproline; RIPA: radioimmunoprecipitation assay; TBA: total bilirubin acid

\section{Competing interests}

The author(s) declare that they have no competing interests.

\section{Authors' contributions}

$\mathrm{CL}$ and PL conceived the study design. CL, LW, GW and GC performed the data analysis. CL and MS drafted the manuscript. CHL and PL revised the manuscript. All authors confirm that the content of this paper has not been published elsewhere and does not overlap or duplicate their published work. All authors read and approved the final manuscript.

\section{Acknowledgements}

This study has been supported by the projects of the National Natural Science Foundation of China (No 90409020 and No 3070 1070), E-institutes of Shanghai Municipal Education Commission (No E03008), Shanghai TCM Clinical Liver Disease Center (No ZX200703).

\section{References}

I. Friedman SL: Molecular regulation of hepatic fibrosis, an integrated cellular response to tissue injury. J Biol Chem 2000, 275:2247-2250

2. Ala-Kokko L, Pihlajaniemi T, Myers JC, Kivirikko KI, Savolainen ER: Gene expression of type I, III and IV collagens in hepatic fibrosis induced by dimethylnitrosamine in the rat. Biochem J 1987, 244:75-79.

3. Bataller R, Brenner DA: Hepatic stellate cells as a target for the treatment of liver fibrosis. Semin Liver Dis 200I, 21:437-45I.

4. Liu P, Hu YY, Ni LQ: On establishing comparative reference system for syndrome classification study from the thingking characteristics of syndrome differentiation dependent therapy. Zhongguo Zhongxiyi Jiehe Zazhi 2006, 26:45I-453.

5. Mu YP, Liu P, Long AH, Du GL, Wang L, Liu Y, Li FH: Study on pathogenesis of $\mathrm{CCl}_{4}$ induced cirrhosis formation in rats based on the recipe used. Zhongguo Zhongxiyi Jiehe Zazhi 2006, 26:344-347.

6. Zhang Q, Liu P, Chen HF, Chen L, Cao SH, Liu Y, Wei JJ, Fang ZH, Wu DZ: Multi-analysis of characteristics of traditional Chinese medical syndrome of hepatocirrhosis. Zhongxiyi Jiehe Ganbing Zazhi 2003, 13:69-72.

7. Arai M, Yokosuka O, Fukai K, Kanda T, Kojima H, Kawai S, Imazeki F, Hirasawa $H$, Saisho $H$ : A case of severe acute hepatitis of unknown etiology treated with the Chinese herbal medicine Inchinko-to. Hepato Res 2004, 28: I6I-165.

8. Yamashiki M, Mase A, Arai I, Huang XX, Nobori T, Nishimura A, Sakaguchi S, Inoue K: Effects of the Japanese herbal medicine 'Inchinko-to' (TJ-135) on concanavalin A-induced hepatitis in mice. Clin Sci (Lond) 2000, 99:42I-43I.

9. Sakaida I, Tsuchiya M, Kawaguchi K, Kimura T, Terai S, Okita K: Herbal medicine Inchin-ko-to (TJ-I35) prevents liver fibrosis and enzyme-altered lesions in rat liver cirrhosis induced by a cholinedeficient I-amino acid-defined diet. J Hepatol 2003, 38:762-769.

10. Inao M, Mochida S, Matsui A, Eguchi Y, Yulutuz Y, Wang Y, Naiki K, Kakinuma T, Fujimori K, Nagoshi S: Japanese herbal medicine Inchin-ko-to as a therapeutic drug for liver fibrosis. J Hepatol 2004, 41:584-591. 
II. Imanishi $\mathrm{Y}$, Maeda $\mathrm{N}$, Otogawa $\mathrm{K}$, Seki S, Matsui H, Kawada $\mathrm{N}$, Arakawa T: Herb medicine Inchin-ko-to (TJ-135) regulates PDGF-BB-dependent signaling pathways of hepatic stellate cells in primary culture and attenuates development of liver fibrosis induced by thioacetamide administration in rats. J Hepatol 2004, 41:242-250.

12. Lee TY, Chang HH, Chen JH, Hsueh ML, Kuo JJ: Herb medicine Yin-Chen-Hao-Tang ameliorates hepatic fibrosis in bile duct ligation rats. J Ethnopharmacol 2007, 109:3 I8-324.

13. Du GL, Liu P, Wang L, Wang CS, Long AH, Mu YP: Relationship between traditional medical formulae and syndrome based pharmacological study of Xiayuxue decoction against porcine serum induced liver fibrosis in rats. Zhongguo Shiyanfangjixue Zazhi 2007, I 3:3-33.

14. Long AH, Liu P, Li FH, Mu YP, Du GL, Wang L: Effect of different ratio of Huangqi decoction on cholestatic cirrhosis in rats. Zhongguo Shiyanfangjixue Zazhi 2006, 1 2:28-30.

15. Mu YP, Liu P, Du GL, Wang L, Long AH, Liu Y, Li FH: Different classic recipes treatment CCL4-induced liver cirrhosis. Progress Nat Sci 2006, 16:1101-1108.

16. Shimizu I, Ma YR, Mizobuchi Y, Liu F, Miura T, Nakai Y, Yasuda M, Shiba M, Horie T, Amagaya S, Kawada N, Hori H, Ito S: Effects of Sho-saiko-to, a Japanese herbal medicine, on hepatic fibrosis in rats. Hepatology 1999, 29:149-160.

17. Scheuer PJ: Classification of chronic viral hepatitis: a need for reassessment. J Hepatol | 99|, |3:372-374.

18. Jamall IS, Finelli VN, Que SS: A simple method to determine nanogram levels of 4-hydroyproline in biological tissues. Anal Biochem 198I, I I 2:70-75.

19. Nakamuta $M$, Ohta S, Tada S, Tsuruta S, Sugimoto R, Kotoh K, Kato $M$, Nakashima Y, Enjoji M, Nawata H: Dimethyl sulfoxide inhibits dimethylnitrosamine-induced hepatic fibrosis in rats. Int J Mol Med 200I, 8:553-560.

20. Jezequel AM, Mancini R, Rinaldesi ML, Macarri G, Venturini C, Orlandi F: A morphological study of the early stage of hepatic fibrosis induced by low dose of DMN in the rats. I Hepatol 1987 , 5:174-I8I.

21. George J, Chandrakasan G: Molecular characteristics of dimethylnitrosamine induced fibrotic liver collagen. Biochem Biophys Acta 1996, 1292(2):215-222.

22. George J, Chandrakasan G: Biochemical abnormalities during the progression of hepatic fibrosis induced by dimethylnitrosamine. Clin Biochem 2000, 33:563-570.

23. limuro Y, Fujimoto J: Strategy of gene therapy for liver cirrhosis and hepatocellular carcinoma. J Hepatobiliary Pancreat Surg 2003 I $0(1): 45-47$

24. Mu YP, Liu P, Liu Y, Li FH, Chen GF, Gu HT, Du GL, Wang L, Wang CS: Inhibiting action of Xiayuxue decoction on hepatic fibrosis at progressive stage in rats and study on the prescription and syndrome. J Tradit Chin Med 2006, 47:215-218.

25. Chena MH, Chena JC: The role of TGF- $\beta$ I and cytokines in the modulation of liver fibrosis by Sho-saiko-to in rat's bile duct ligated model. I Ethnopharmacol 2005, 97:7-13.

26. Araki N, Noda T, Ogawa K: Cytochemical studies on the effect of intraperitoneal and oral administration of a traditional Chinese medicine (Sho-saiko-to) on the D-galactosamineinduced hepatic injuries of rats. Acta Histochemica Cytochemica 1988, 21:439-453.

27. Shiba M, Shimizu I, Yasuda M, li K, Ito S: Expression of type I and type III collagens during the course of dimethylnitrosamineinduced hepatic fibrosis in rats. Liver 1998, 18:196-204.

28. Li D, Friedman SL: Liver fibrosis and the role of hepatic stellate cells: New insights and prospects for therapy. J Gastroenterol Hepatol 1999, 14:618-633.

29. Wang L, Liu P, Mu YP: Study on TCM recipe and syndrome of DMN-induced hepatic liver fibrosis in rats. J Tradit Chin Med 2006, 47:929-932.

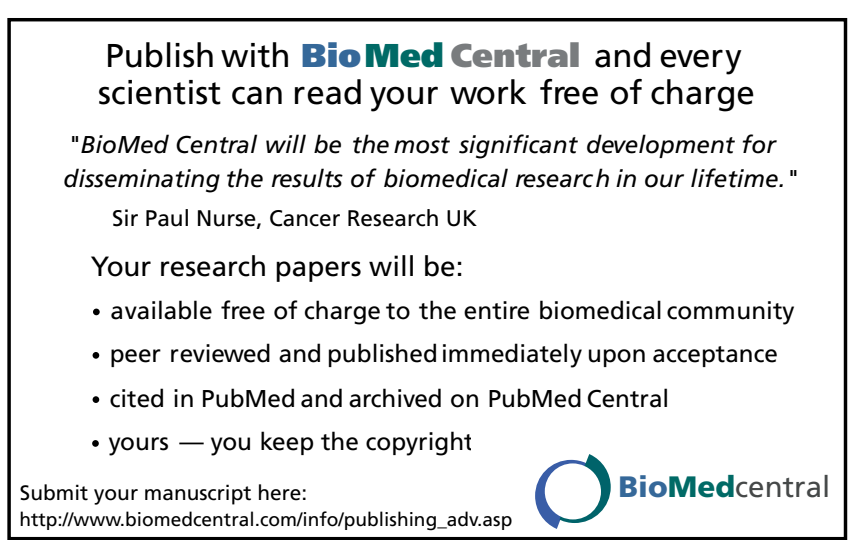

